\title{
Authority as an interactional achievement: Exploring Deference to Smart Devices in Hospital-Based Resuscitation.
}

Menisha Patel PhD, BA, Research Associate

Wolfson Building, Department of Computer Science

University of Oxford, OX1 3QD

01865270550

menisha.patel@cs.ox.ac.uk

Mark Hartswood PhD, MSc, BSc (Hons) (Corresponding Author)

Senior Researcher, Wolfson Building

Department of Computer Science, University of Oxford, OX1 3QD

01865270550

Fax : $+44(0) 1865283532$

mark.hartswood@cs.ox.ac.uk

Helena Webb PhD, MA, BA (Hons), Senior Researcher

Wolfson Building, Department of Computer Science

University of Oxford, OX1 3QD

01865270550

helena.webb@cs.ox.ac.uk

Mary Gobbi, PhD, MA (Ed), Dip N, Dip NEd, RGN, Professorial Fellow (Nursing Education) Faculty of Health Sciences, University of Southampton,

SO17 1BJ, 02380598270

mog1@soton.ac.uk

Eloise Monger PhD BSc(Hons) RGN FHEA, Lecturer in Critical Care Nursing Room E3013 Building 67, Faculty of Health Sciences, University of Southampton

02380597992

em@soton.ac.uk

Marina Jirotka PhD, MSc, BSc, Professor of Human Centred Computing

Wolfson Building, Department of Computer Science

University of Oxford, OX1 3QD

01865270551

marina.jirotka@cs.ox.ac.uk 


\section{Abstract}

Over the years, healthcare has been an important domain for CSCW research. One significant theme carried through this body of work concerns how hospital workers coordinate their work both spatially and temporally. Much has been made of the coordinative roles played by the natural rhythms present in hospital life, and by webs of mundane artefacts such as whiteboards, post-it notes and medical records. This paper draws upon the coordinating role of rhythms and artefacts to explore the nested rhythms of the Cardio-Pulmonary Resuscitation (CPR) protocol conducted to restore the proper heart rhythm in a patient who has suffered a cardiac arrest. We are interested in how the teams delivering CPR use various 'smart' assistive devices. The devices contain encoded versions of the CPR protocol and are able to sense (in a limited way) the situation in order to give instructions or feedback to the team. Using an approach informed by ethnomethodology and conversation analysis (EM/CA) we analysed video of trainee nurses using these devices as they delivered CPR in dramatized training scenarios. This analysis helped us to understand concepts such as autonomy and authority as interactional accomplishments, thus filling a gap in CSCW literature, which often glosses over how authority is formed and how it is exercised in medical teams. It also helps us consider how to respond to devices that are becoming more active in that they are being increasingly imbued with the ability to sense, discriminate and direct activity in medical settings.

\section{Keywords}

Authority, Autonomy, Resuscitation, Team Work, Smart Devices.

\section{Introduction}

This paper explores the use of two Smart Devices to assist with the resuscitation of patients who have suffered a cardiac arrest, and examines in close detail how they have the capacity to both disturb and assist existing practices. We adopt an in-depth, qualitative approach to examine the practical use of these devices in simulated resuscitation scenarios. Our analysis demonstrates that these devices play an interactional role in the ongoing simulation, and, that in some instances an asymmetry is constructed in which the device might be seen to exert 'authority' over teams of nurses delivering basic life support. This opens up questions around autonomy and authority that are important but rarely directly addressed within the CSCW literature, particularly in the context of healthcare teams.

As with other walks of life, Smart Devices are becoming increasingly pervasive in medical settings. Automated External Defibrillators (AEDs) and Smart Infusion Pumps are already commonplace. Increasingly sophisticated devices are being rolled out, such as Smart Beds that can monitor a patient's vital signs and intelligently adapt to their posture. There are several drivers for the increasing use of Smart Technologies in healthcare. For example., automation is seen to provide a way of meeting the predicted increased demand for care of the rapidly aging population ${ }^{1}$. Smart Devices promise also to improve the effectiveness of healthcare, or promote wellbeing via the paradigm of ubiquitous health (e.g. Orwat et al., 2008). Harm reduction provides a further motivation to develop intelligent systems able to monitor drug doses and check for adverse interactions (e.g. Rothschild et al., 2003;

\footnotetext{
${ }^{1}$ http://www.extremetech.com/extreme/207130-terapio-autonomous-medical-robot-can-assist-nurses
} 
Ammenwerth et al., 2008). Finally, commercial interests presumably play a role in driving attractive product enhancements as an incentive to renew and upgrade existing equipment. While there are undoubtedly positive benefits to innovative Smart Devices, there are also potential negative effects in relation to patient safety, the integrity of teamwork, professional role and identity, and the values attached to care (Cummings and McGowan, 2011; Elgin and Bergero, 2015).

The first device that we investigate is an Automated External Defibrillator (AED) which is able to detect and correct an abnormal heart rhythm by delivering an electric shock. AEDs were originally designed to support a layperson in delivering CPR in public places, but are now increasingly replacing manual defibrillators in hospitals. The second is a prototype Smart Watch application, which was developed within our project to provide feedback about the rhythm and correct depth of chest compressions as CPR is administered. The context for our study is a simulated hospital setting where multidisciplinary teams interact to perform resuscitation as part of a training simulation for student nurses. The 'patient' is a robot known as SimMan $\mathbb{R}^{2}$. We use this setting to examine in detail the interactions between the nurses, a more experienced practitioner and the two key Smart Devices.

Team resuscitation in hospitals is a pressured and emotionally charged activity where the timing and quality of interventions is critical to patient survival. Resuscitation involves enacting a standardised United Kingdom and European protocol (Resuscitation Council 2015) and is typically conducted by ad-hoc teams formed at the moment a cardiac arrest is discovered. Delivery of the protocol requires intricate levels of coordination between team members, who have to establish, maintain and renegotiate roles spontaneously throughout the procedure. Effective leadership within these ad-hoc teams has been associated with improved CPR outcomes. While the protocol has an underlying rhythm, it is also complex and includes elements of repetitive physical actions, monitoring, evaluation and problem solving. The clinical decision making processes promoted by the CPR algorithm may necessitate further concurrent activity, some driven by further algorithms, to address underlying or associated clinical problems; for example, if a patient is discovered to be hypothermic, diabetic, or septic. Machines, equipment and devices are integral to the performance of hospital-based resuscitation. They feature in the team choreography in complicated ways, sometimes serving more as a prop to help maintain the flow of activity, and at other times more prominently as an actor exerting authority over the situation.

By taking a fine-grained qualitative approach our analysis is able to identify the intricate ways in which team members incorporate the Smart Watch and AED into their ongoing activities. In this paper we illustrate two phenomena of particular interest and discuss their implications for practice. In the first we observe a scenario in which a group of student nurses orient to a Smart Device in ways that treat it as hierarchically superior to their own actions and likely understanding of the resuscitation protocol. This orientation has the effect of diverting the students from delivering a complicated protocol that they are only just beginning to get to grips with and creating trouble in the ongoing activity. In this instance there are missed opportunities for the Device to provide 'appropriate' cues to assist the activity as a competent protagonist in the successful accomplishment of resuscitation. In the

\footnotetext{
${ }^{2}$ http://www.laerdal.com/gb/doc/86/SimMan
} 
second phenomenon of interest a nurse practitioner playing the role of a doctor in the simulation arrives on the scene and overrules the instructions voiced by the AED. A different kind of hierarchy is constructed and all the team members ultimately interact with the Device in a very different way than observed in our first scenario. The nurses' asymmetrical relationship with the Device is challenged; new forms of interacting with it are opened up and legitimised, perhaps to be deployed by the nurses in future encounters.

Our analysis of the data is informed by insights from ethnomethodology and conversation analysis (EM/CA). We then draw on these findings to discuss broader concepts concerning the enactment of authority and autonomy in the delivery of CPR. This includes the role played by Smart Devices or 'active artefacts' (Carbitza and Simone, 2009) in the construction of asymmetries in the setting. We conclude with design principles for devices, protocols and training to reduce the counter-productive disruptive potential of the devices.

\section{Background: Rhythms, Temporality and Teamwork}

Over the years, healthcare has been an important domain for CSCW research (Fitzpatrick and Ellingsen, 2013). One significant theme carried through this body of work concerns how hospital workers coordinate their work over space and time. Much has been made of the coordinative roles played by the natural rhythms present in hospital life, and by webs of mundane artefacts such as whiteboards, post-it notes and records.

At first glance, many features of this earlier work are visible in the work of emergency resuscitation. The sequence of activities specified by the CPR protocol occur in a series of nested rhythms that also play an organising role in that there are 'natural pauses' where information may be gathered, tasks initiated and roles exchanged. Artefacts play an important coordinative role too. Hospital based resuscitation involves a wide range of equipment, drugs and other artefacts which are pre-organised and pre-arranged in a Standard Operating Configuration (Bardram and Bossen, 2005) so that they are easily and conveniently available as and when the protocol demands they be used. But there are a number of differences too. The rhythms of CPR operate over much smaller spatial and temporal scales than the rhythms considered by Reddy et al. (2006), with each cycle of compressions and breaths taking only 30 seconds to complete, and with an average resuscitation event lasting only 20 minutes in total. Moreover, previously considered rhythms, such as ward rounds or lab result availability are an emergent part of the hospital life-world, and their production is more or less taken for granted. With CPR, the production and perpetuation of the rhythm, and in particular, the fidelity to the protocol, is a crucial feature of resuscitation and critical for the survival of the patient.

Work focussing specifically on resuscitation practices has considered issues of temporality, for example, Kusunoki and Sarcevic (2015) consider the requirements of temporal awareness in a trauma resuscitation team. They find that perception of time may be dilated or compressed during the fast-paced activity of resuscitation and identify how team members orient to temporal awareness for staging of procedures, assessing team performance and the overall progress of the resuscitation attempt. Dynamically formed adhoc medical teams responding to urgent or emergency situations have also been considered in the CSCW literature in terms of their constitution and communication requirements. Lee 
et al. (2012) observe that such teams, as well as being 'ad-hoc', are also 'loosely formed', with team members joining and leaving while the team is active.

Badram (2000) analyses the temporal coordination of interdependent activities occurring within surgical teams. One aspect he draws out pertains to dynamic teamwork, which, is 'characterised by continuous synchronisation of the many actions and actors involved, according to the concrete conditions in the work' (pp. 165). This focus on co-located actors working synchronously within a team centred upon the patient comes closer to the situation of resuscitation within our study. In this context, Bardram identifies three types of coordination activities: Communicative coordination (via utterances or gestures); Instrumental coordination (based upon the awareness of activities of others) and Scripted coordination (via a shared schedule or protocol which all orient to).

In a similar vein, Pine's (2012) study of choreography in midwifery identifies discrete 'choreography practices' - Moving (body movements, glances etc.), Synching (interleaving e.g. documentation with other tasks), Juggling (simultaneously performing a number of tasks), Prepping (anticipating future tasks and organising the current workspace to make these easier), Collaborating (mutual assistance between nurses and an ongoing re-division of labour), and Triaging (continuous assessment of the importance of the separate tasks that need to be attended to).

However, aspects of leadership and authority are, for the most part, absent in many of the depictions of team coordination and choreography present in the CSCW literature. This is strange as it risks implying that the team members play an equivalent role in performing or initiating activities at all times. It is as if, within these studies, we are continually pointed to the synchronised performance of the orchestra playing, but never shown the work of the conductor who leads. One exception is Sarcevic et al. (2011), who has explored the nature of leadership in the context of trauma teams, observing different models of shared leadership within their extensive ethnographic video corpus of trauma resuscitations.

Whilst we can find in our case study several of the coordinative practices outlined above, we go beyond these to explore how the tasks to be accomplished are given their relative importance so that priority may be assigned, and how determining this relative importance can be seen as part of the interactional accomplishment of authority within these settings.

\subsection{Perspectives on Professional Authority and Protocols}

Whilst issues around authority, and related concepts such as autonomy, have not been frequently discussed in the CSCW literature, they have been discussed elsewhere, particularly in relation to protocols and guidelines (such as the CPR protocol) that serve to guide practice. Concepts of autonomy and agency are also important within the nursing profession, as nursing continues to seek its own professional identity to overcome being defined as a 'service profession' to doctors (e.g. Weston 2008). New technologies are frequently perceived as a threat to professional authority, particularly where they enhance surveillance (Epling et al., 2003) or mandate clinical process (Kent et al., 2015). However, such fears are not new and have been expressed before in relation clinical guidelines, evidence-based medicine and decision-aids. Yet in practice these innovations have become 
widely established without significantly undermining prized and guarded professional authorities. One reason for this, Timmermans and Kolker (2004) argue, is that there never existed a single unambiguous set of power relationships for protocols and guidelines to rule over. Instead, there is a whole mess of temporally evolving, heterogeneous, institutional and professional sources of clinical knowledge, and disparate foundations for legitimate decision-making, all available simultaneously within the clinical professions (ibid). From a relational view of autonomy (Donchin 1995; McDonald 2002), guidelines enter this nexus as yet another resource for action that may preserve existing authorities, as much as displace them, which can be seen in how nurses utilise protocols as a resource to legitimise their own decision-making (Manias and Street, 2000).

While a singular target for any authoritarian effect of guidelines remains elusive, so does the locus of practice as a site of authority:

'The illusion of total bureaucratic supervision and control, prevalent in too many tales (both dystopian and utopian), is a chimera: the multiplicities and contingencies embedded in the workings of a protocol cannot themselves be controlled.'

(Timmermans and Berg, 1997)

Timmermans and Berg (1997) show how the active participation of practitioners is essential to animate guidelines so they are able to influence practice. Thus the very authority that guidelines seem to threaten is actually part of what allows them to work in the first place, precisely because the clinician's discretionary interpretation is needed to adapt the letter of the guideline to the non-compliant everyday circumstances in which the guidelines would otherwise struggle to function (ibid).

In summary, this prior work suggests that we should see authorities as relational (shaped by circumstance and context), as multiple (jostling together in a dynamic that evolves over time) and as mutually interdependent (one authority depending on the exercise of another for its realisation). We return to these issues later in the paper to consider what happens when protocols are asserted by Smart Devices via a range of interactional capabilities, as opposed to being represented on paper in a docile way.

\section{Resuscitation and Training}

In this section we detail resuscitation events incorporating AEDs in particular, and we offer a contextual background to some of the key factors associated with resuscitation practices. Cardiac arrest is a relatively rare event, and is one of only a few spontaneous events where the actions that immediately follow have a significant influence on whether an individual lives or dies. The events which cardiac arrest triggers, the resuscitation attempt, generally begins with one individual who summons a rapid response from a team with expertise. This may be by calling an ambulance - usually by a lay bystander, or in hospital by calling the cardiac arrest team - usually by a nurse. As well as summoning expertise, this initial call leads to the arrival of equipment, most notably an AED. The initial response with limited personnel and/or expertise is called Basic Life Support (BLS), which involves the rescuer performing chest compressions and mouth to mouth breathing. Those performing BLS are known as first responders and in a hospital setting usually comprise of local clinical 
personnel based in the vicinity. BLS usually commences with one person and is ideally undertaken involving three people subject to their availability: one to maintain the airway, one to undertake compressions and a third person to gather and use locally based equipment.

The next step on the continuum is Advanced Life Support (ALS) which takes place when a team of experienced specialists convene around the patient. The core BLS actions (compressions, airway support and if available defibrillation) continue until they are augmented by more advanced resuscitation techniques. The ALS team is drawn from resuscitation experts who undertake their normal clinical roles within the hospital until an emergency call is received. The team usually comprises a designated anaesthetist, medical practitioner with resuscitation expertise, a technician and a senior nurse. As a consequence, these teams are ad hoc (Roberts et al., 2014), they are not well formed and members may not even know each other. The ALS team are unlikely to know the first responders. The team may arrive at different times into an unfamiliar environment to manage the resuscitation attempt, and co-ordinate both themselves and the environment's staff (Miller et al., 2012). Typically, someone from the patient's supervising medical team will be called to advise on the impact of any concurrent health conditions that could influence the patient's management. As can be seen, several people will be engaged in the resuscitation, each one with different roles, levels of expertise and arrival times.

As the membership of the team around the patient expands numerically during the course of the resuscitation, but specifically with the arrival of the ALS team members, then the management of the individuals present becomes increasingly important to ensure that their skills are deployed appropriately within the context of the recommended guidelines and the patient's condition. Hence, the UK 2015 guidelines advise that BLS training for staff who regularly perform CPR should include 'non-technical' training that incorporates skills in communication and team behaviours. Leadership skills, and how to be an effective member of a resuscitation team, are included within these guidelines for those undertaking Advanced Life Support training. For any hospital based resuscitation event, the ALS training instructs the ALS certified staff to not only assume the leadership role upon arrival, but also to nominate and articulate their designation to the first responders present. ALS certification is not profession specific, and therefore a nurse, if the first to arrive, may take initial leadership and then pass on the responsibility to an anaesthetist or senior physician when they arrive. Normally ALS medical staff take over the leadership role because their scope of practice includes the ability to prescribe and initiate treatments beyond that of other healthcare professionals.

The potential for problems with the performance of these ad hoc teams is understood, and adherence to complex algorithms is challenging and errors are frequent (Yamada et al., 2015). National standardised training in ALS was established in 1993 to provide structure to the resuscitation attempt as a means of control. So, the 'script' for undertaking ALS has been well-defined but the roles which individuals take in practice and how they perform these in the moment is largely unestablished. There are different ways of approaching this problem (Wacker and Kolbe, 2014; McLanders et al., 2015), but it is clear that no-one has yet undertaken the work required to provide more solid clarification of issues in enacting 
the script. In particular, there appears to be little work analysing events before the arrival of the ALS team.

We have already mentioned that successful resuscitation events require effective team relationships, which include effective task management, leadership and communication skills (Castelao et al., 2013). Certain styles of leadership are favoured in such teams. These styles of leadership termed 'Horizontal' or 'Lighthouse' leadership are where the leader remains practically disengaged- taking an overview of the ongoing situation, asking questions and giving precise instructions, but at the same time is responsive to the capabilities of the team and their ability to self-organise and conduct tasks without external direction (Schenarts 2007; Cooper and Wakelam, 1999).

Critical to patient survival is early defibrillation, reducing what is known as 'hands off time' (namely breaks in the cycles of cardiac compressions), with the quality and rhythm of the compressions and airway support being other crucial factors. While there are existing validated and reliable measures of individual and team performance in resuscitation (see McKay et al., 2012), and the UK 2015 Resuscitation guidelines specify the roles of the respective team members, the use of the AEDs and other devices, little attention has focussed specifically upon the human factors related to the effect of the devices upon performance and vice versa, yet empirical studies of AED use in hospitals reveals they contribute to significant 'hands-off' time during resuscitation (Smith et al., 2011). It is noticeable in the systematic review of team co-ordination that Castelao et al. (2013) undertake and the study by Andersen et al. (2010) concerning barriers to teamwork in CPR, that while the importance of sub tasks and resources are mentioned, there is no reference to the impact of the technological devices on team co-teamwork ordination or effectiveness, aside from device failure. Interestingly, Section 2 of the UK 2015 guidelines draws attention not only to the important role of social media and innovate technologies in improving patient outcomes, but also encourages their evolution, development and analysis.

It is known that there are tensions caused by the paradox created by AED voice command adherence during basic life support, the advice to have minimum interruptions to chest compression (Resuscitation Council 2015), and the delays or interruptions to performance caused by the voice commands themselves (e.g. Monsiuers et al., 2005). Similarly, the extent to which Smart Devices are attuned to the competence level of the rescuer and the context of the resuscitation appears neglected in the literature. Ironically, because basic life support is conducted prior to the arrival of the experts and multi-disciplinary teams (MDT), it is only through simulation or in vivo observation that the effect of the devices can be fully noted.

\section{The Smart Technologies Investigated}

In this study, the two smart technologies used were a model of AED commonly used in public and hospital settings, and a SmartWatch prototype developed within our project, to assist with the compression component of the resuscitation procedure.

Prior to the development of the AED, the only way someone could receive defibrillation was by ALS trained professionals using unrestricted defibrillators requiring professional 
discretion, guided by the relevant algorithm. The ALS professional could determine the frequency, strength and appropriateness of defibrillation. AEDs were developed to reduce the time from collapse to defibrillation by enabling a layperson to initiate an automatic process that could not be altered. This meant defibrillation could take place in public spaces and everyday environments such as retail, sports venues and schools. However, it soon became apparent that the AED could be used by first responders in hospitals, who were also unable to administer unrestricted defibrillation. Similarly, this had the effect of reducing the time from collapse to first defibrillation. However, the restrictions of the AED mean that the ALS competent practitioners could not use their professional judgments with the AED. Hence, some models of AED were developed with the capacity for a manual override to give flexible administration of defibrillation when ALS certified staff arrived. Currently, healthcare students do not have the training nor scope of practice to override manually an AED. However, they are trained to try to reduce 'hands off' compression time. The AED measures and analyses a patient's heart rhythm and then if it is required, provides an appropriate shock to the patient's heart in order to restore a normal heart rhythm. Given the primary protagonist is a non-professional user, assumed to have little medical knowledge, the design of the AED intends to provide a highly authoritative and linear structure for what is required to be done when using it, in order to resuscitate the patient. The process is now described.

The AED device comprises a control box with three simple written and pictoral instructions which guide the rescuer to (1) open the device; (2) turn it on and (3) follow the voice instructions. In our study, the sequence of voice prompts can be summarised in this way:

1) Apply electrode pads to bare chest of patient

2) Insert pad connector next to flashing light on AED device

3) Do not touch patient

4) Analysing heart rhythm- Pause

a) No shock advised continue CPR OR

b) Shock advised Do not touch the patient

5) Charging

6) Do not touch the patient

7) Deliver shock now

8) Press the orange button now (to deliver the shock)

9) Shock delivered

10) Pause

11) Start CPR if necessary

The first two stages are part of the procedure for initially applying the AED within the resuscitation algorithm. Once the AED is applied, the sequence then runs cyclically every three minutes from step 3 which interrupts the rescuers resuscitation activities of cardiac compression and airway support. For adults, the current sequence of compressions to breaths is 30: 2 . It was to aid the acquisition and performance of compression competence that the second Smart Device was developed.

The second smart technology used was a Smart Watch developed within our project to facilitate and potentially improve the provision of compressions during CPR. This sensor based Smart Watch is designed to be worn by the nurse who is responsible for physically 
conducting compressions in a resuscitation attempt. When used, the watch will measure the speed, depth and number of compressions that the nurse provides. The purpose of this technology is firstly to deliver key credentials of compressions in the ongoing situation to facilitate the information needs of participants. For example, it may indicate to a nurse that her compressions are too shallow, allowing her to reshape her practice and thus, providing 'better' treatment to the patient. Importantly, such information related to compressions can allow nurses to understand, through physical practice, how to improve and develop their skills in deliverance of compressions for future situations. Nurses are informed through theory of what an appropriate cycle of compressions should involve, and trained using mannequins. In this situation, it may be difficult to understand or get feedback regarding the exact shape of the compressions they produce and how they align to what is required by their training algorithms. This watch provides such feedback, which could have potential benefits for aligning the nurses to the standard of what a 'good' cycle of compressions may feel like in practice. The technical details of the development and pilot deployment of the SMART Watch are outlined in Grünerbl et al. (2015).

\section{The Case Study: Smart Devices in Simulated Resuscitation}

The study was set in the Clinical Skills Laboratories in the University of Southampton Faculty of Health Sciences. This simulated ward environment has six bed spaces with associated controllable cameras and hanging microphones which capture and store audio and video feeds, similar to a CCTV recording system. Adjacent to the ward there is a smaller operations room where the trainers, or at times trainee nurses, can observe training simulation exercises occurring in the main ward. This allows for observation of simulations without being disruptive, and the activity can be recorded to allow for training purposes or retrospective reflection on materials following a training exercise. During training sessions, the role of the patient is played by a technological simulator called SimMan ${ }^{\circledR}$ which can display vital signs (e.g. pulse, breathing, blinking, speech) that are controlled remotely via a dedicated laptop. SImMan $₫$ is a mobile, portable, interactive robot mannequin designed to enable healthcare teams to practise pre-programmed emergency or technical situations which required teamwork, clinical decision making and procedural skills. SimMan ${ }^{\circledR}$ has lifelike anatomy and clinical functionality. Concurrent management of the Simulation enables reactive consequences to be initiated in response to team member actions (e.g. example cessation or return of breathing, retching sounds and changes to the heart rhythm). The computer logs of SimMan $®$ were available for analysis, together with the 360 degree audio visual camera material.

\subsection{Methodology}

Our approach to exploring these issues has been to examine in a detailed way the use of these devices in a simulated practice environment. The main corpus of our data was collected during several sessions of training simulations over four days at Southampton University. 


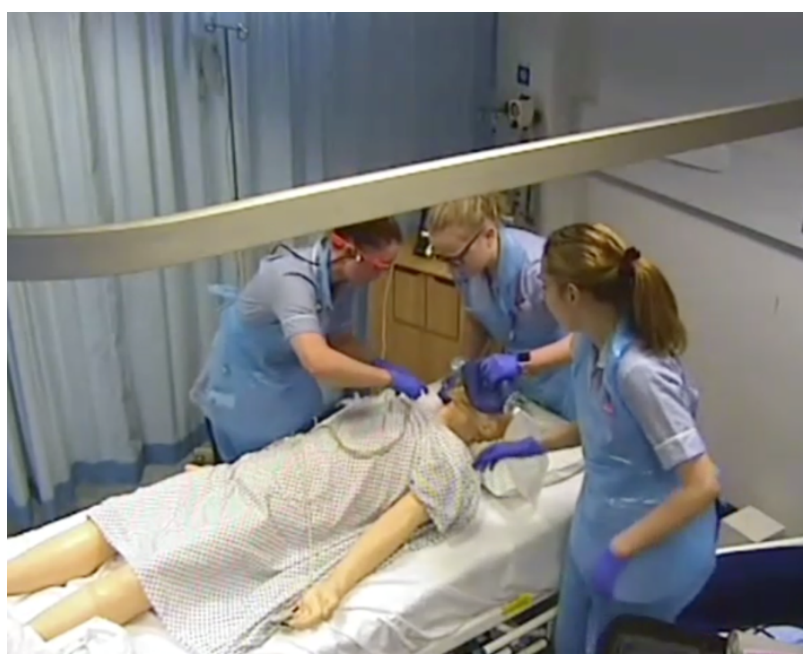

Figure 1: trainee nurses interacting in the simulated practice environment

Student nurses were recruited from the year 2 cohorts of the Post Graduate Diploma in Adult Nursing and Adult Bachelor of Nursing. Permission to approach the students was obtained from their Programme Leads and the students were invited to participate through a brief presentation during a Progamme Management Session . Five days before the training simulations, interested students were provided with an introductory letter, Participant Information Sheet and Consent Form. Eighteen student nurses were recruited to participate in the study. The students expected to engage in simulated practice scenarios - similar to those they have undertaken in their Programmes. While they had received training in basic life support and the use of AEDs during their first year, and undertaken approximately 1500 hours of real clinical practice at this stage of their training, very few students would have participated in a real resuscitation event. The 'trainers' and staff 'actors' (e.g. the doctor) were recruited by approaching Faculty Resuscitation trainers directly.

\subsection{Ethics and governance}

The study protocol was approved both by the University of Southampton Faculty of Health Sciences Ethics Committee and the Central Research Ethics Committee at the University of Oxford. Particular attention was paid to ensuring that student participants understood the issues of anonymity and consent as they applied to the video data when subsequently disseminated in the public domain. Students received feedback on their performance for their own personal development as well as for the purposes of the research. Students were encouraged to provide feedback on the process of the study and to be co participants in the study with respect to the use to the technology aids.

\subsection{Data collection}

We conducted a total of six simulation sessions lasting around thirty minutes each. The procedures for collecting, reviewing and analysing video data were informed by those set out in Heath et al.'s (2010) description of video- based fieldwork and the University of Southampton's bespoke Protocol for Research and Education/Ethics into Virtual Interactive Practice (Monger and Gobbi, 2006).

In each training session, a group of up to four trainee nurses were provided with a scenario surrounding the condition of a patient through an initial briefing by a trainer. 
This would emulate what is termed a 'handover' between teams at a hospital, where necessary information for the care and responsibility of a patient is passed between different practitioners. Following this, the nurses would start to work collectively towards caring for the patient with minimal intervention from the trainers. The condition of the patient would be deteriorated by the trainers (positioned at a distance, often behind a curtain within the ward) at some stage in the scenario, to necessitate the use of CPR. In the majority of sessions, the nurses were asked to use the AED when resuscitating the patient. At some point in their scenario, a doctor played by an experienced ALS certified practitioner, also intervened in the situation. Through the scenario, the students have the opportunity to experience the arrival, assertion and enactment of leadership made by an ALS practitioner. Following this session, the nurses were debriefed by trainers. This is important for nursing education, allowing them to reflect upon and thereby develop their skills in a quasinaturalistic situation.

Our observational material was available in the operations room through CCTV, and collected via the audio-visual infrastructure embedded within the simulation ward, allowing for the collection, observation and consideration of high quality video data.

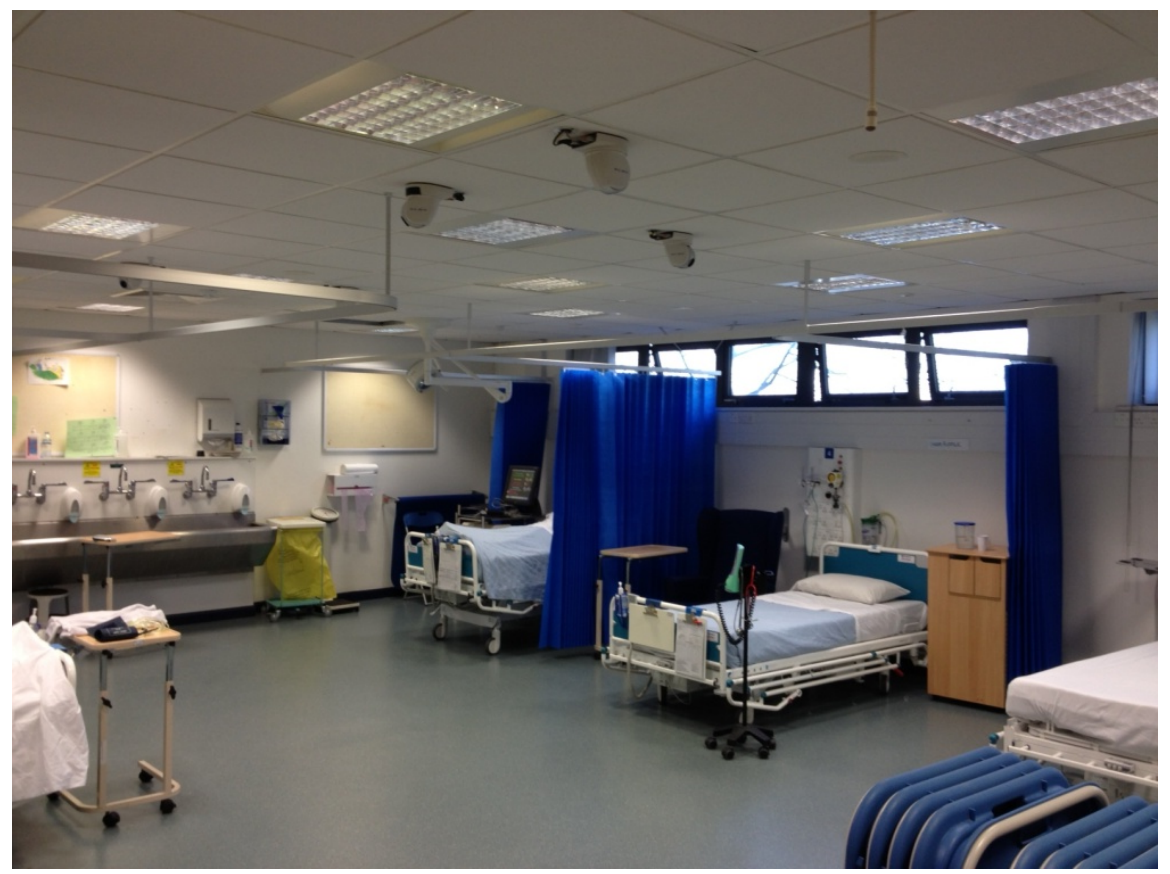

Figure 2: the simulation ward

Having collected the video data we followed an inductive approach and reviewed the entire corpus to identify areas of interest relating to the ways in which the participants in the simulation sessions interact with other and with the technologies being used in their tasks. Our analytic interest lay in how the activities taking place in the sessions unfold in a moment-by-moment, sequential manner. We therefore sought to identify sequences of interest within the wider data, and focussed on instances in which a Smart Device is drawn in to the ongoing task and in which it has the potential to cause some kind of disruption to the activities being undertaken by the human participants. We made a collection of these instances and analysed them to a high level of qualitative detail - in particular drawing on the insights of ethnomethodology and conversation analysis (EM/CA). These methodological 
approaches are based on the fine-grained analysis of naturally occurring interactions and help us to understand the ways in which the particular outcomes of simulation sessions and more specifically the capacity for smart devices to have a positive or detrimental impact -are not pre-determined but rather are contingent on the interactions taking place within them. Of particular use was work describing the character of institutional interactions (for instance, Drew and Heritage, 1992). This work highlights the relevance of institutional identities (nurse, doctor, patient etc.) to the accomplishment of tasks at hand and the tendency for asymmetries and constraints on interaction to exist in institutional settings. For instance, in medical settings it is very rare that practitioners talk about their own (rather than their patient's) illnesses and in surgical ones it is typical for hierarchies to emerge in which experienced practitioners are more likely to speak and be listened to than junior ones at points of emergency.

Members of the research team were also present during the simulation sessions and were able to collect fieldwork data to supplement the video recordings. These took the form of ethnographic observations plus informal interviews with the trainee nurses, trainers and also the developers of one of the smart technologies- the Smart Watch- that was used to treat the patient.

This analysis revealed patterns of action and interaction occurring across the dataset, which highlight salient issues in the configuration of socio-technical environments where smart technologies are used. This paper discusses two data fragments which illustrate the key themes occurring across the wider data collection. Our analysis provides an empirical basis for wider conceptual discussion of authority in the sessions. We use it to discuss the ways in which authority appears to be constituted at a local level, through moment-by-moment interaction.

\subsection{Analytic Findings: Teamwork and Nurse Resuscitation}

In this section, we present some of our key analytic findings relating to the use of Smart Devices in simulation sessions and their capacity to (positively or negatively) influence the conduct of medical training tasks. We focus on two exemplar data fragments and two types of Smart Device: the automated electronic defibrillator (AED) and the Smart Watch.

\section{Fragment 1: Smart Watch}

In this data fragment a group of trainee nurses are using the AED and Smart Watch technologies in order to facilitate the resuscitation of the (simulated) patient. At the start of the fragment we observe that the Smart Device appears to be incorporated smoothly into the conduct of the resuscitation. As the activity goes on however, a problem occurs when the Device measures compressions asynchronously with the compressions actually being conducted. We present a detailed analysis of the interactions occurring in this data fragment in order to show that:

- the ongoing activities in the setting are accomplished through the interactions of those present;

- this includes interactions between the nurses and the nurses' interactions with the Smart Device; 
- in this particular scenario the nurses orient to the Device in ways that treat it as hierarchically superior to their own actions and likely knowledge of the resuscitation protocol;

- the construction of this interactional asymmetry results in the technology appearing to shape the conduct of the simulation and its outcome.

The transcript (see Transcript 1, below) shows verbatim the spoken utterances that occur (including those of the AED). It also includes some features of Jeffersonian transcription (Jefferson 1972) standard in EMCA to detail the occurrence of pauses - shown as numbers in brackets - and micropauses of below 0.3 seconds- shown as (.). Unclear talk is shown in brackets and square brackets are used to indicate overlap between participants. We also use screen grab images from the video recording to highlight key visible details - such as the physical position of the nurses and patient. Arrows are used to link the image to the relevant point in the transcript.

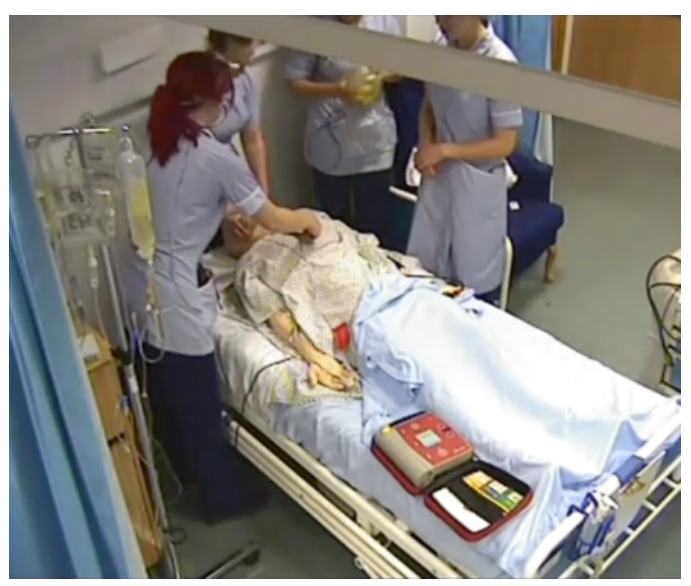

Figure 3:

The nurses are labelled on the transcript according to their position around the bed; N1: is operating the AED; N2: is based at the head of the bed; N3: is responsible for maintaining the patient airway; N4: is tasked with undertaking compressions at relevant junctures; $A E D$ : refers to voice of the AED.

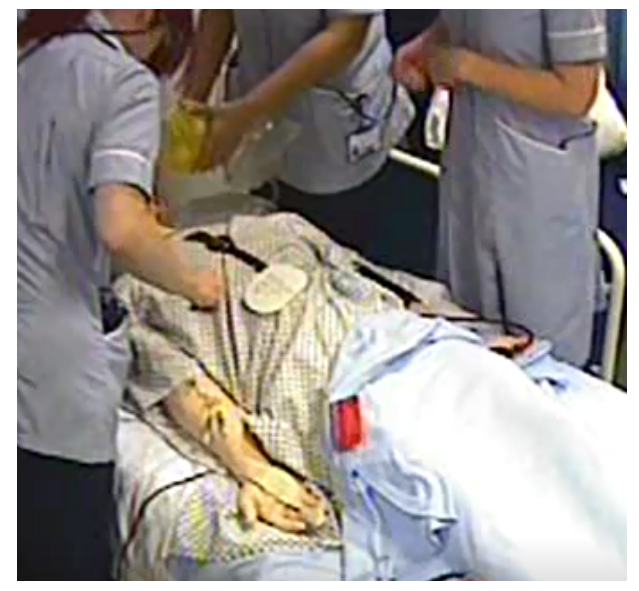

Figure 4:

Illustrates the positioning of the Smart Watch, which is difficult to make out on the subsequent images accompanying transcript 1.

As the fragment begins, the AED has taken a measurement of the patient's heart rhythm and consequently instructs that a shock should be delivered to the patient. This shock is administered through the press of a button on the AED. Once it has been given, compressions can take place - and the Smart Watch can be used to help regulate the pace of this procedure. 


\section{Transcript 1}

1. AED: deliver shock (.) shock delivered

$(0.6)$
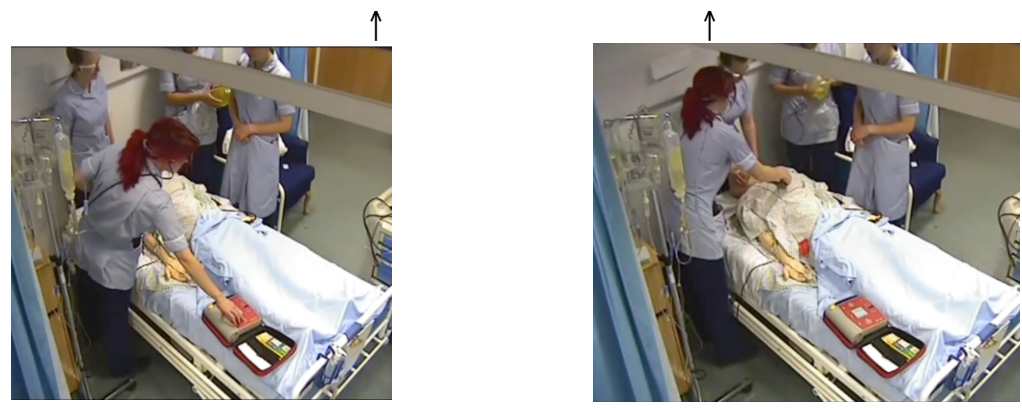

2. AED: Pause

$(0.8)$

3. AED: if needed start CPR

$(0.2)$

4. N4 :
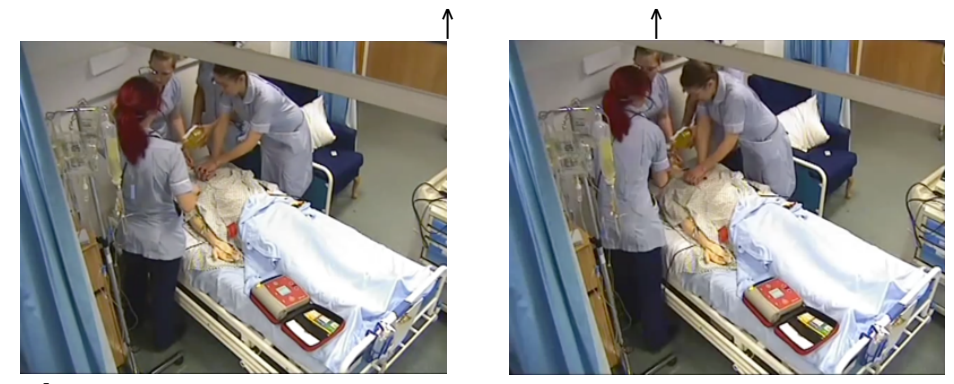

[11 lines omitted]

16. N4: okay oh

$(\cdot)$

17. N4: oh

$(0.4)$

18. N1: its uhm

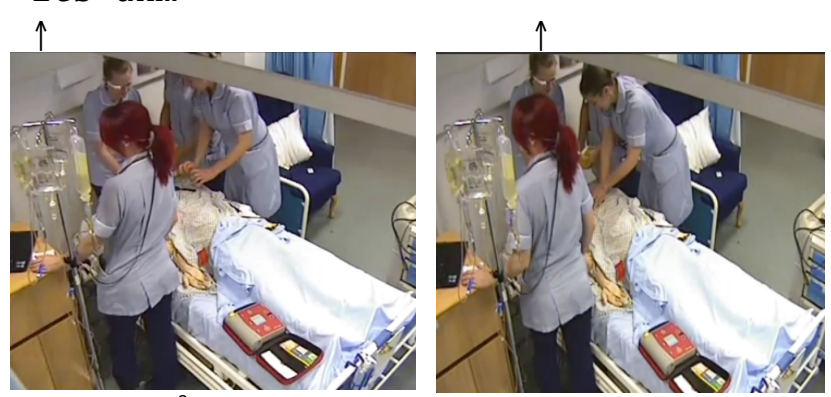

19. N2:

( ${ }^{3}$ its like moved)

it went to seven)

(.)

20. N1: okay

\footnotetext{
${ }^{3}$ A bracketed utterance indicates that it was not fully clear what was being said at that moment, and instead there is an interpretation of what is likely to have been said given available audible data. Where an utterance is entirely inaudible, brackets will enclose no text at all, and instead mark that the occurrence of the utterance rather than details of what that utterance was or an interpretation of this.
} 
In this short fragment (lasting for 71 seconds in total) the two technologies become relevant to the ongoing interactions in the setting and the accomplishment of the task at hand. The AED instructs a shock to be delivered to the patient (line 1 ) and this is completed by one of the trainee nurses - N1. N1 then places the Smart Watch on the chest of the patient. The AED advises to start CPR 'if needed' and another nurse, N4, begins compressions. At this point we can make some observations on the ways in which the participants interact both with each other and the technologies present when accomplishing their activities.

In administering the shock and beginning compressions the nurses draw on their institutional knowledge of the AED sequence, conducting the different steps required at each appropriate point. The particular ways in which these steps are accomplished are contingent on phenomena within the setting itself. We can see the activities occurring in this fragment as taking the form of sequences of action, with the conduct of one action oriented to by the participants as projecting a next relevant action to follow. For instance, $\mathrm{N} 1$ treats the AED's utterance 'deliver shock' in line 1 as making relevant the pressing of the button on the AED - not only in terms of performing the action but also in its timing so that it occurs directly after the utterance. She then treats the pressing of the button as making relevant the placing of the Smart Watch on the patient's chest. In turn this placement projects the involvement of the watch as relevant to the next action that will occur - the compressions, which are then conducted by N4. A sequence is unfolding in which one action builds on another and activities are accomplished via collaboration between participants. We observe that the AED is oriented to as a (non-human) participant in this setting; its utterances are listened to and responded to. In fact, its utterances in lines 1 and 3 are treated as instructions, with the nurses responding to them immediately. So the inclusion of this Smart Device appears to result in a kind of hierarchy or asymmetry between actors.

We also observe that the Smart Watch is made relevant to the ongoing sequence through the actions of the participants. When N1 places it on the patient's chest she projects its presence as relevant to what is about to occur - the compressions. She places it in a particular way so that its face can be easily seen by N4, who will be conducting the compressions. With this action N1 displays sensitivity to the ongoing task at hand, the particular ways in which the task will be achieved and the needs of her co-participants. As a result the Watch becomes available to be used at the appropriate moment and without disrupting the ongoing procedure in any way.

In the opening phase of this data fragment both the AED and Smart Watch are integrated into the activities being undertaken by the nurses. This appears to be achieved in a relatively seamless way that does not disrupt the clinical tasks being undertaken. Furthermore, we can observe that the AED is being treated as an asymmetric, non-human participant, able to issue instructions. Its treatment is asymmetric because its instructions are given priority over the nurses own knowledge of the protocol. As the fragment continues the Smart Watch also becomes attended to as playing a kind of asymmetric role but in this instance it becomes problematic to the accomplishment of the medical task at hand.

After the AED's utterance in line 4, N4 begins compressions on the patient - once again treating the device as legitimately able to issue instructions. According to the standard procedure, N4 is required to undertake a cycle of 30 compressions, made in a steady 
rhythm. As she completes her first compression, N1 moves her right arm to the Watch (Figure 5); as she completes the second, N1 taps the face of the Watch. What has happened is that the Watch has not turned on and is therefore not measuring the compressions as it is supposed to. N1's actions attempt to switch the Watch on and continue (Figure 5) as N4 carries out the third and fourth compressions. In fact, N4 takes a longer pause between the third and fourth compression than previously - an action which appears designed to aid N1's attempts to switch on the Watch. In this way both N1 and N4 treat the Watch as contingent to the ongoing task and the absence of its measurement as problematic. Their attempts to resolve the problem in turn disrupt the rhythm of the compressions.
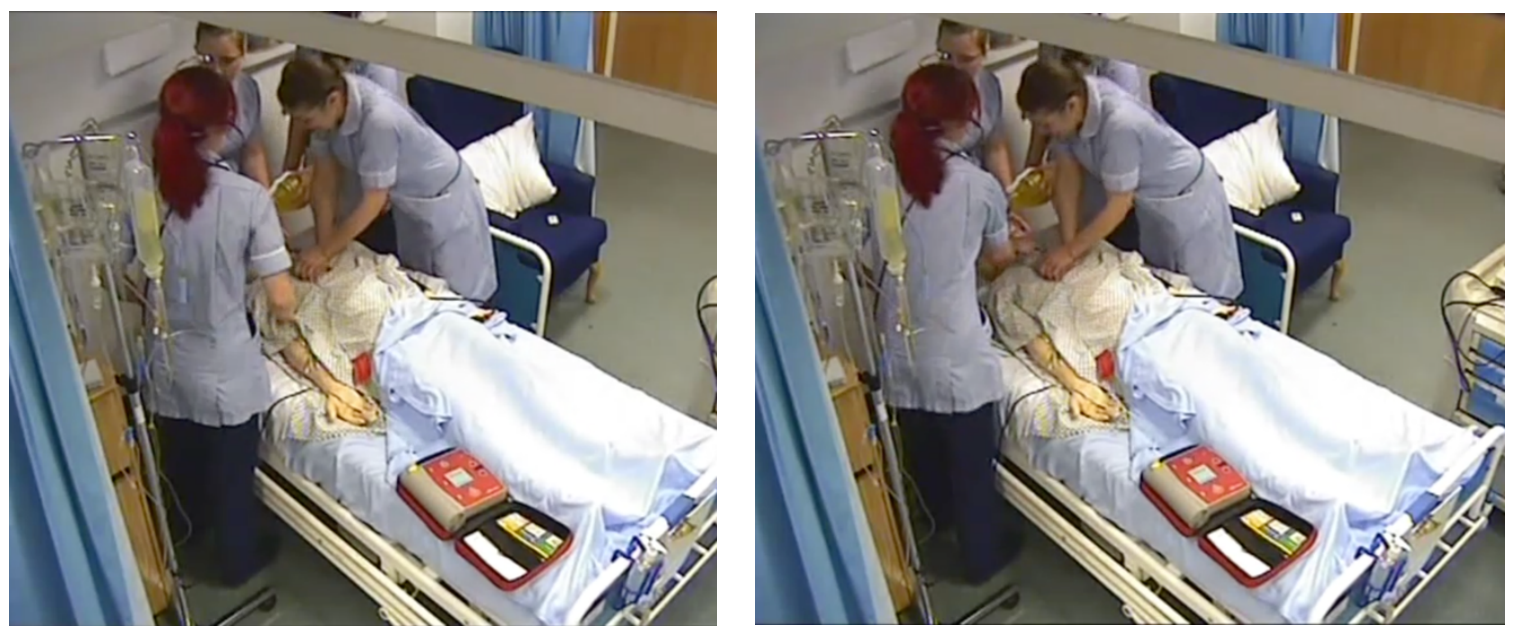

Figure 5a and 5b: N1 moves right hand towards watch in an attempt to switch it on

This disruption continues over the fifth and sixth compressions with $\mathrm{N} 1$ continuing to attempt to switch the Watch on and N4 producing a longer than usual pause between compressions. The Watch has been turned on by compression 7 and $\mathrm{N} 4$ resumes a faster and steadier rhythm. The Watch is now operating but is asynchronous with the actions of $\mathrm{N} 4$. This results in further problems once $\mathrm{N} 4$ has completed the cycle of 30 compressions. At the end of the cycle $\mathrm{N} 4$ moves slightly upwards but remains leaning forwards towards the patient with her hands clasped together in the 'CPR position' (Figure 6). She then moves slightly further upwards and moves her hands into a kind of claw shape (Figure 6). She visibly maintains readiness to carry out compressions even though the cycle of 30 has been completed. She whispers to N3 (on her right), who then appears to point to the screen of the Watch. N4 moves back down towards the patient, clasping her hands together in a way that suggests she is about to perform further compressions. As she does so N2 shifts her gaze away from the screen to her right and down to the Watch. The attention of all four nurses is directed towards the Watch and the particular misalignment that has occurred between the compressions it has measured and the compressions that have been performed. N4 then gives the patient further compressions - going against standard protocol but following the requirements of the Watch. 

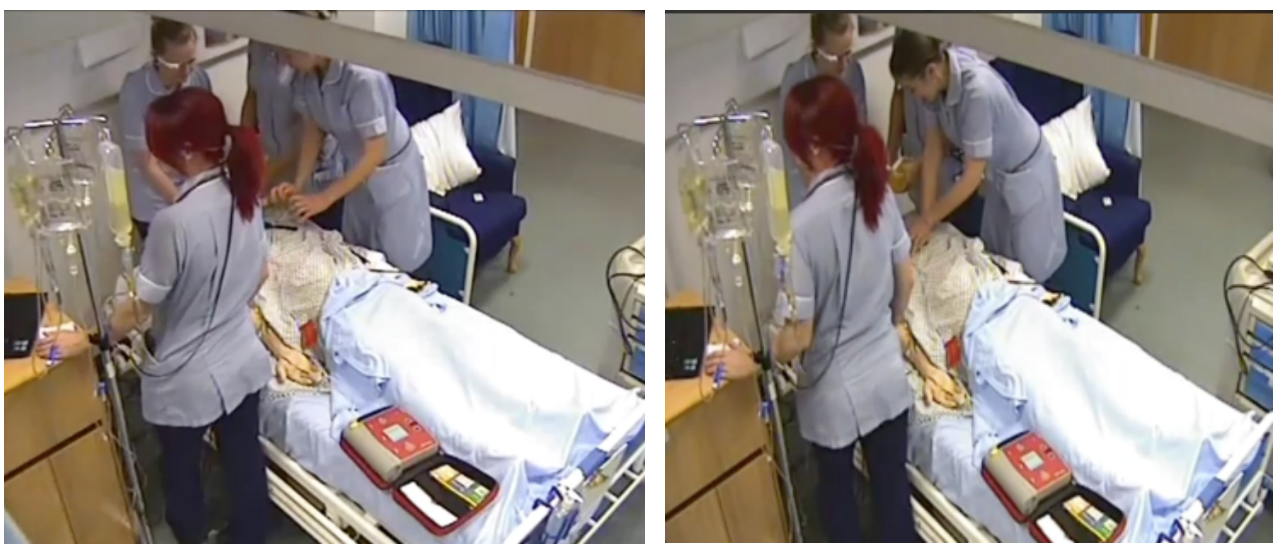

Figure 6a and 6b: N4 moves back into undertaking compressions despite having completed a cycle of 30

In the first phase of this fragment we observed the Smart Devices being integrated seamlessly into the activities of the simulation. However, analysis of the second phase reveals that the Smart Watch becomes problematic when it measures compressions asynchronously with the compressions actually being conducted by N4. The nurses attend to this misalignment as problematic in their visible and spoken actions and their attempts to rectify the problems are disruptive to the conduct of the compressions and result in a breach of standard CPR procedure. In this environment, something as apparently mundane as not being able to switch the Watch on causes what could potentially be a serious obstacle to the smooth completion of the resuscitation procedure. We can observe that the start of the compressions appears to be a particularly vulnerable moment in which this kind of obstacle might emerge, given the importance of establishing a rhythm in synchrony with the measurements of the Watch.

It is important to note that it is not simply the presence of the Watch or the occurrence of the problem switching it on that determines that the disruption to the procedure occurs. Rather, the disruption emerges through the particular ways in which the nurses attend to the Watch - and made continued attempts to switch it on. The nurses respond to the directions of the Watch ahead of their own formal knowledge of the procedure, treating it as having a hierarchically superior status in the ongoing activity. In other scenarios the technology is not treated as having this status and this asymmetry does not appear in the interaction. In fragment 2 asymmetrical relationships in the setting emerge in a different way, with both the nurses and the AED complying with instructions given by a doctor.

\section{Fragment 2: Experienced Practitioner}

In this second data fragment a group of four nurses is once again conducting a simulated resuscitation procedure facilitated by the AED device. In this instance an experienced nurse practitioner is present, playing the role of a doctor in the simulation and therefore referred to as 'Doctor' in the transcript. The Doctor issues instructions to the nurses that contradict the utterances of the Device. Our analysis illustrates that: 
- the Smart Device once again takes a role as a non-human participant in the ongoing activity;

- however, a different kind of hierarchy emerges as the Doctor contradicts the utterances of the device;

- the nurses present orient to the instructions given by the Doctor ahead of those issued by the Device;

- the Doctor ongoingly manages and maintains that asymmetrical role through embodied interaction at key moments in the situation.

The fragment lasts for 27 seconds; as it begins N1, positioned to the left of the doctor (Figure 7), is placing pads pads on the lower torso of the patient so that the AED can begin to measure the heart rhythm. N3 is giving compressions.

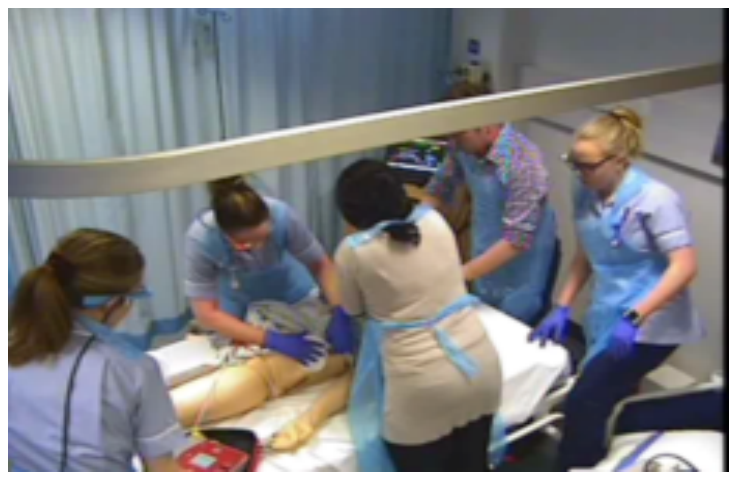

Figure 7:

The nurses are labelled on the transcript according to their position around the bed; $\mathbf{N} \mathbf{1}$ : is placing the pads on the patient; N2: is based on the right of the doctor at the head of the bed; N3: is responsible for compressions and is an experience qualified nurse; N4: is operating AED; AED: refers to voice of the AED; D: refers to the doctor

\section{Transcript 2}

1. AED: Analysing heart rhythm $(0.9)$

2. AED :

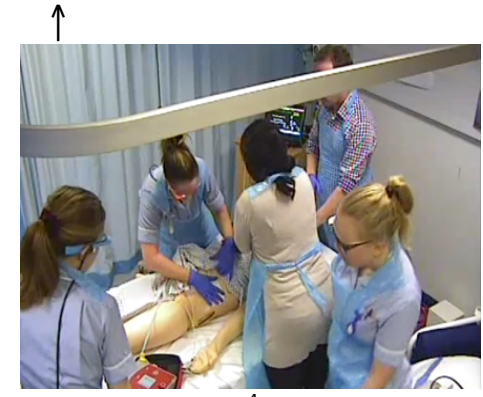

3. D: =okay=

4. AED: =touch the patient (.)

${ }^{4}$ the 's' symbol marks a very rapid transition from one utterance to the next. 
Authority as an interactional achievement

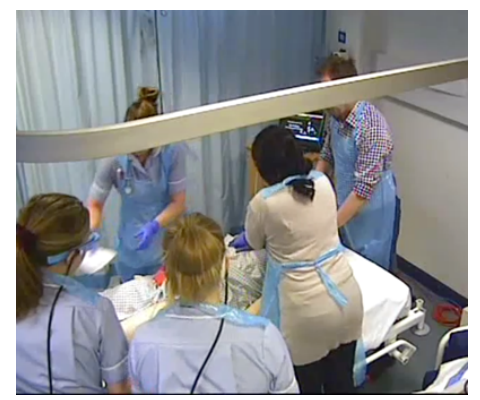

5. N4 :

okay stand by

6. D : stop for a moment while

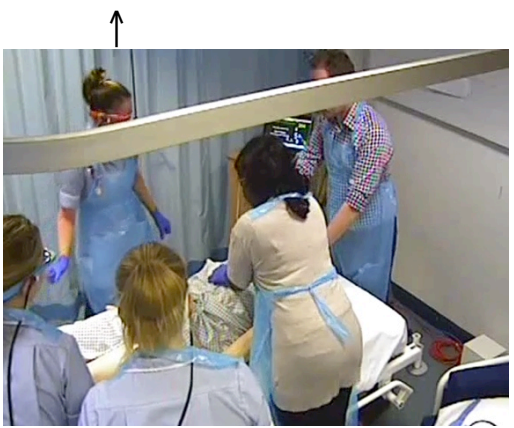

it analyses its gonna

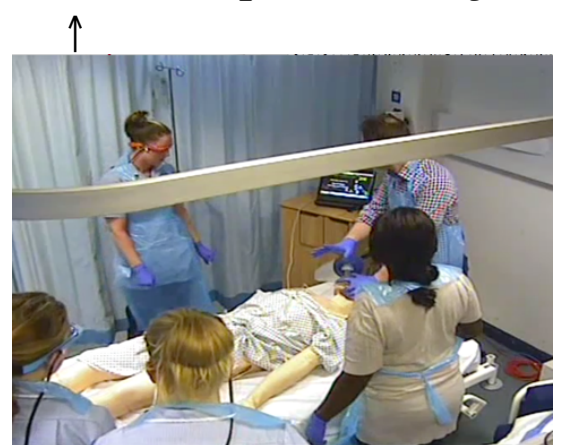

$(2.2)$

7. AED: shock advised=

8. D: =start- you start $C P R$ for the moment $(0.4)$

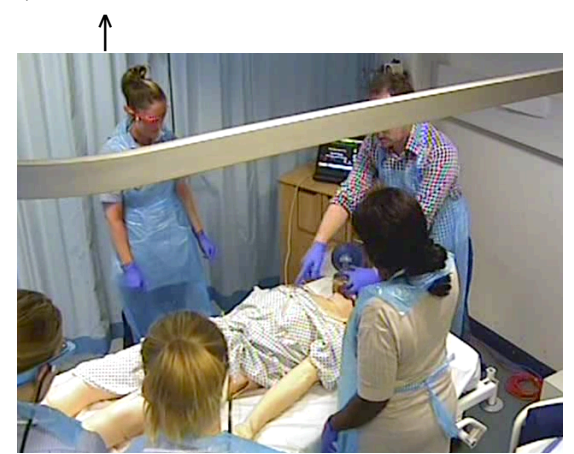

9. AED :

10. D: =let it charge

$$
\text { charging= }
$$

$$
(\cdot)
$$

11. AED: stand clear of the patient

12. N4:

okay stand

13. D :

$$
\text { let it charge }
$$


14. D: let it charge $(0.4)$

15. AED: deliver shock now

16. D: stand clear

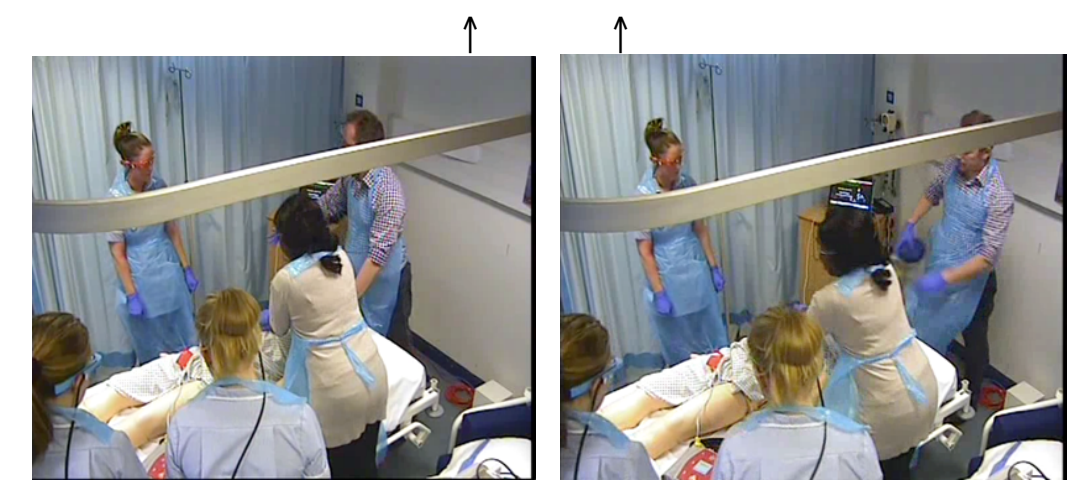

17. AED: press orange button (.) shock delivered

$(\cdot)$

18. D: okay start $\mathrm{CPR}=$

19. N3: =okay=

20. AED: =pause

$(0.6)$

21. AED: if needed start CPR

Once again the AED here takes a role as non-human participant in the resuscitation procedure. In line 1 it makes an announcement that it is analysing the heart rhythm of the patient. As it does so, N1 completes placing the pads on the patient's lower torso and N3 continues compressions.

In lines 2-4 the AED says 'do not touch the patient'. As with fragment 1 we can see this as the AED issuing an instruction. However in this instance, this instruction is not treated as requiring an immediate response by all of the participants in the setting. As the AED says 'Do not' N1 removes the pad wrapper from the patient's leg and moves away. She anticipates the instruction from the AED and complies with it, even whilst it is being produced. By contrast N3 remains giving compressions. It appears that she is drawing on her own formal knowledge of the procedure as more immediately relevant than the instruction of the device.

In line 5 N4 says 'okay stand by'. This reformulates the utterance from the AED not to touch the patient and thereby aligns with the instruction it has given. It can also be seen as an attempt to encourage others - for instance N3 - to align with it. The Doctor begins a turn in overlap with her line 6 . He speaks in a loud voice and says 'Stop for a moment while it analyses it's gonna'. As he says this he raises his right arm to a height just above the patient's chest and brings it palm down, visible to all the nurses and close to N3. N3 stops giving compressions. As he completes his utterance, both N1 and N3 take a step back, away from the patient.

By this point three potential instructions have been given: one by the AED, one by N2 and one by the doctor. It is only the Doctor's instruction that receives immediate compliance from all the nurses. It appears that a different kind of asymmetry is emerging in this 
interaction: at least one nurse (N3) treats the AED as not necessarily taking precedence of her own formal knowledge of the procedure and the Doctor is treated as holding a hierarchically superior status than both the nurses and the AED. It is worth noting that N3 is an experienced, but not ALS (advanced life support) certified, practitioner and this may have some bearing on how she interacts with the AED. Doctors of course are typically understood to have a high status (due to their level of expertise) in medical settings so we can observe here that institutional and professional identities are being played out to construct this particular hierarchy. It is also useful to note the particular way in which the Doctor gives his instruction - with a raised voice and a marked bodily gesture that is visible to all those present. We can see these as a practical enactment of both the Doctor's formal hierarchical and ALS leader status and, combined with the nurses' response, as the moment-by-moment achievement of 'authority' in the medical setting (see Figure 8, below).

As the fragment continues we see further ways in which the actions of those present constitute an asymmetry in which the Doctor has a hierarchically superior status than the AED and the nurses. In essence, the Doctor gives instructions that contradict those of the $A E D$ and the nurses comply with them rather than responding to the smart device. The Doctor's actions create a misalignment with the device - rather than in Fragment 1 where the misalignment is precipitated by the technology itself - and treat this misalignment as unproblematic for the successful accomplishment of the procedure. We can see this again as instances of the operation of 'authority' in interaction.

As he completes his instruction 'Stop for a moment while it analyses it's gonna', the Doctor looks towards the screen on his right, which displays information about the patient's vital signs. This gives him an opportunity to analyse the patient's condition, even though this is what the AED is currently doing. He treats his own independent analysis as relevant in this situation and the AED's analysis as (potentially) not enough on its own (see Figure 8).

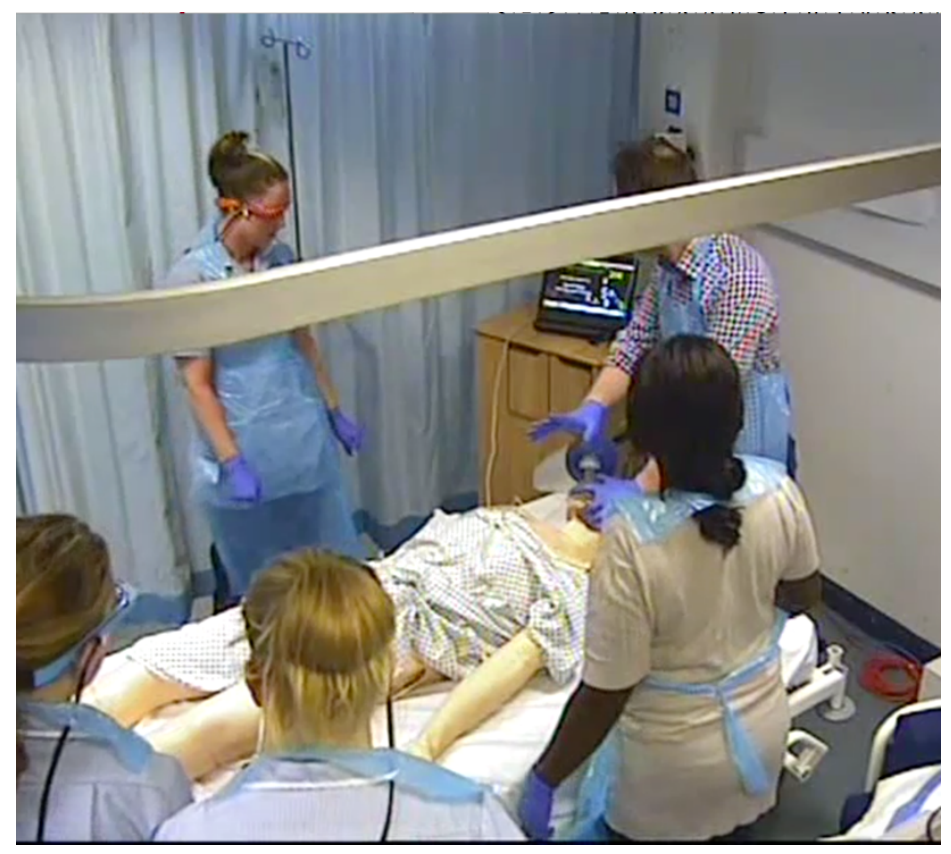

Figure 8: the doctor holds his hand over the patient to indicate the pause in compressions 
The AED then advises that a shock is given to the patient. (line 7). The nurses remain still; in contrast to Fragment 1, they do not appear to treat the AED's utterance as an instruction requiring immediate compliance. The Doctor turns away from the screen and points his hand towards the patient's chest. He then gives his own instruction in line 8 'start -you start CPR for the moment', directed towards N3. This instruction is in direct contrast to the AED's advice to shock the patient. N3 moves into position and begins compressions. She thereby aligns with the instruction of the Doctor ahead of the advice of the AED. The other nurses also slowly shift forwards towards the patient, also aligning with the Doctor's direction.

From lines 9-11 the AED first states that it is charging and then advises those present to 'stand back'. This is standard procedure as the device will deliver a large electric shock to the patient that can be dangerous to others. The Doctor however directs the nurse to maintain compressions during the time period when the device in charging. During this time, N3 continues giving compressions. Her actions align with the instruction from the Doctor rather than the AED.

As the AED utters the word 'shock' (in line 15) the doctor says 'stand clear' first sweeping his hand back towards the wall, and then physically moving backwards towards the wall behind him. Here he gives an instruction in addition to the AED but one that aligns with it rather than contrasts it. As he has previously overruled the advice of the AED we could consider this additional instruction a necessary indication to the nurses about what to do, as at this point it might be unclear to them whether or not they should align with the device. Once again the Doctor uses an embodied gesture to accompany his instruction and this plays a role in the embodied exercise of his asymmetrical position in this scenario. On the word 'clear', N3 finishes her compression and she and the other nurses move away from the patient, thereby aligning with his instruction giving.

With everyone in the room positioned away from the patient at an appropriate distance, N4 presses the button to deliver a shock to the patient and the AED then confirms that shock has been given (line 17). Following this, the nurses and doctor remain in their positions at a distance from the patient. A few moments later the doctor gives a new instruction 'okay start CPR' and then moves his left arm to the neck of the patient to check the patient's pulse. His movement indicates that it is now safe and relevant to move back to the patient. N3 aligns with this instruction by moving back to the patient and beginning compressions. Whilst she is giving them, the AED issues further instructions, first to 'pause' and then to begin CPR. So this is a further instance in which the Doctor has drawn on his formal knowledge rather than the AED to determine appropriate action to take and in which his instructions are oriented to by the nurses as requiring compliance ahead of the AED.

Analysis of fragments 1 and 2 reveals some important insights into the use of smart devices in medical training scenarios. Firstly, they show that the role played by these devices is not pre-determined but is rather contingent on the moment-by-moment unfolding of activity in the setting. It appears that devices can be seamlessly integrated into ongoing activities (first phase of fragment 1) creating an opportunity for them to facilitate the task at hand. They can also have the potential to be disruptive, with misalignments between the technology and the conduct of the activity (phase two of fragment 1) creating problems that need to be resolved. In both fragments there were times when participants' own formal knowledge of 
the procedure contrasted with the instructions given by the technology, however the ways in which these ruptures were resolved differed. In fragment 1 the nurses followed the advice of the technology whereas in fragment 2 the Doctor led the nurses to disregard some of the device's instructions. We can argue that the institutional hierarchies and asymmetries inherent to medical settings play a role in constituting how misalignments between formal knowledge and input from the technology are dealt with. In fragment 1 the nurses' responses to the Smart Watch created an asymmetry in which the advice of the Watch was treated as superior to their own knowledge. By contrast in fragment 2, the Doctor's actions treated his own knowledge and expertise as superior to that of the AED. In their actions the nurses complied with this, creating a scenario in which the Doctor issued directions for the nurses to follow and the device was at times overruled. Consequently, there are important issues around autonomy and authority to consider when discussing the use of Smart Devices in these kinds of training simulation. This is discussed next.

\section{Discussion}

In the fieldwork analysis above we saw how CPR is conducted in a highly technologised environment, involving a multi-focal choreography of individual practitioners, items of equipment, devices, and the patient itself. In this discussion we firstly touch upon the importance of the rhythmic accomplishment of CPR and some issues surrounding the practicalities of this accomplishment. Following this and very much related to some of the initial issues that we draw upon, we consider the asymmetries exhibited at different moments between the Smart Devices, the junior and senior practitioners, and how these asymmetries are constitutive of the authority of the actors and hence their ability to take the lead in the aforementioned choreography.

The time-critical rhythms that scaffold and underlie the process of resuscitation are crucial to its undertaking. The successful resuscitation of a patient, in a large part, depends on the repetition of a sequence of actions that have to be reproduced with high fidelity. This broader rhythm includes the different phases that the participants move through, including the establishment of CPR, the arrival of a senior practitioner and bringing CPR to a close. Our fine-grained analysis shows that within this broader rhythm is the nested rhythm of CPR, comprising moment-by-moment rhythmic elements - including the pace and depth of compressions, the switching between breaths and compressions, and the periodic pauses which are taken to assess the patient. The accomplishment of the multiple rhythms is constituted of sequential interactional, where an action is shaped by what occurred prior to it, and goes on to shape the next action. Within these, the fluid interrelation of and between these multiple rhythms plays an important role in coordinating the practitioners- both individually and collaboratively, and their temporal interrelationship to the space they work within, and the tools and artefacts that they use.

There are certain features of the interaction that provide opportunities for the rhythm to be disturbed. In the transitory point between each of the broad phases and even the momentby-moment action, junctures exist whereby there is the chance that a relevant next action is delayed or not initiated at all. A key finding that emerged and that shall be considered in more detail as we move through the discussion, is that the collaboration of the different 
participants in the setting is sensitive to certain asymmetries between them. These asymmetries are visible in the manner in which interactional cues are produced by an actor, and how the cues of fellow actors and technologies are responded to. For instance, important to the undertaking of CPR is to minimise the 'hands off' time, that is the time away from the cyclic rhythm of compressions and provision of air to a patient. We see that the more experienced practitioner orients to minimal time away from this rhythm, for example, avoiding stringently abiding by the long pauses that the AED attempts to embed within the situation. Quite differently, the nurses are highly sensitive to the cues of the AED and the smart watch, thus increasing the 'hands off' time and disrupting the overall rhythm of the procedure, and instead deferring responsibility to the technologies. So whilst the smart watch provides 'a beat' to the encourage the fluency of compressions, in this instance it proves to be disruptive, especially when it provides stray cues which the nurses orient to as a priority, instead of the patient. Also, when oriented to as the authority in the situation the AED serves to hinder the temporal progression of the CPR for longer periods than are necessary when used by less experienced practitioners. The differential knowledge of what technologies do, and experience of using them appear to play a part in creating asymmetries, which impacts how and how well the procedure of CPR is undertaken, and the ongoing hierarchy within the situation.

Our data shows normative status hierarchies being played out within the resuscitation simulations. Although we argue that what we are witnessing is how those hierarchies are being reproduced or accomplished, rather than simple acts of deference based on internalised responses to formal professional designations. Here we follow Schneider's ethnomethodological analysis of 'power':

'Power, in this view, is not a discrete entity that is somehow conferred on people or offices by virtue of their position in an organizational hierarchy. It is, rather, something that must be accomplished over and over again in every social interaction by those who would say and have it said about them that they have power'.... 'Power is not something that one can ever have; it can only be accomplished through access to interactional resources that allow one to have one's reality claims accepted as the facts of the matter.' (Schneider 2017)

This is not to say formal status designations are unimportant, but we do need to see how they are brought to life within an interactional frame. Status is certainly significant within the resuscitation protocol itself, which codifies specific designations and appropriate patterns of deference. Thus, the protocol grants a nurse with ALS training a higher authority than a junior doctor. In fact, an ALS responder is taught to 'declare' themselves on arrival to legitimate their authority - thus the protocol and associated training are a source of 'interactional resources' that those assuming authority may draw upon.

For this paper, the idea that power (authority) is '...accomplished by access to interactional resources' (ibid) is a key point. So when the doctor enters the scene, then it's not so much his designation as a doctor that grants his authority (he's not actually, he's an experienced ALS-trained nurse playing a doctor), it is that he is able to deploy certain 'interactional resources' that are not available to the other participants to the resuscitation. Although his designation (and expected arrival) provides an important cue for the other participants to 
orient their interaction to him as a doctor, this orientation will only be sustained if he ongoingly deploys the interactional competencies commensurate with his status. This explains how authority can both be something that is usually roughly aligned to organisational hierarchies, but also (somewhat) fragile and contingent and open to the possibility of being reordered, particularly locally. This situation is reminiscent of Circourel's observation that authority assigned to diagnostic information relied upon the credibility of its 'sources', which need not align with institutionally sanctioned hierarchies, but depended instead upon a continual informal social (re)assessment of that credibility amongst local networks of clinicians (Cicourel 1990). In an analogous way, we suggest that the capacity of clinicians to display authority themselves, and manage situations where may be multiple or competing authorities, depends heavily upon interactional competencies gained through experience or training, which may only loosely align with the status normally associated with professional roles.

The doctor's exogenous experiences, accrued by virtue of his training and his many years of experience in delivering advanced life support, provide him with vital resource, both for transcending the framing that the AED imposes on the situation and for exerting his authority over the human actors. He deploys complicated combinations of interactional resources, including tone of voice, timing, gesture, the 'content' of his utterances, and the spatial positioning he adopts, all of which put constraints on the interactional possibilities available to the other participants, particularly if they are to continue to collaborate in reproducing this status hierarchy. For example, taking over the role of airway management puts him in the 'director's seat', and a role with enough 'hands off' time to enable the use of extended gestures. The hand gesture he deploys to hold the resuscitation (figure 8) claims the space over the patient's body, limiting the range of responses possible for the nurses that still acknowledge the doctor's authority. His utterances are formulated of clear instructions without any room for discussion or dispute, and we see him assert his position by instructing the nurse undertaking compressions to do so, overriding the AED, and also when he interjects as another trainee nurse is attempting to coordinate the room. He clearly maintains a coordinating role in the room through temporally sensitive embodied interaction, that is shaped by prior interaction, and shapes next action. It is not that one resource is always used, more that the use of resources and the manner in which they are used is very much tailored and sensitive to the moment-by-moment interaction and contingencies of the situation.

Whist the doctor's interactional resources have been acquired from prior experience and training, this episode provides opportunities for the nurses to acquire interactional resources of their own. When the legitimacy of the AED is challenged, the doctor's actions become a resource for the trainee nurses, for whom his actions serve as a demonstration of how the CPR protocol can be more aggressively delivered if the authority of the AED is overruled. At the same time, this display provides new legitimate grounds for overruling the AED. Although such a lesson may not be entirely assimilated following a single training event, the potential for the nurses to now reassess how they respond to an AED in a resuscitation event is quite evident.

So a key question then is what interactional resources does the technology possess and how is this shaping the unfolding sequence of actions? For example, the Smart Watch 
interacts by displaying a count of compressions. This constrains the interactants to complete the compressions if the logic of using the watch is to be preserved. There is a sort of accountability that the watch creates for the nurses in the performance of the compressions - after all, compliance with the protocol is the effect that the watch is designed to achieve. Similarly, the authoritative tone of the defibrillator, and use of imperative statements 'Stand Clear!' again demands a certain response in turn in order that the legitimacy of the AED is ongoingly ratified. The problem for the both the watch and the defibrillator is that their embodiment of the protocol is not sensitive to context - the AED does not know that it has been deployed in the hospital, not a public space; and the watch does not know that it has become misaligned with the rhythm of CPR. To have utility in helping nurses to reproduce rhythms of CPR, particularly in a hospital context, these artifacts need to admit human discretion as to when their 'advice' should be adhered to or disregarded.

\subsection{Acquiring interactional resources}

There is a striking asymmetry of the junior nurses in relation to the technology whereby the junior nurses tended to treat the instructions of the AED and Smart Watch with greater legitimacy than their own interpretation of the protocol. While the doctor's authority is in some sense 'fully formed', the nurses are very junior and still very much in the process of acquiring the interactional competences that will support their being able to act with authority. Here we draw on some of our wider ethnographic observations that reveal how the student nurses are developing and exploring the boundaries of their authority within the institutions of medicine. That the nurses are novices is visible in their interactions, including the way that they look for external cues, and express hesitancy, invite and anticipate direction from others. The nurse trainers, whilst observing the sessions remotely, commented frequently on the 'body language' of the nurses, their level of alertness and engagement, and the way that they adopted or failed to adopt specific roles in delivering CPR. The trainers looked specifically for a hierarchy to emerge within the team, whereby one of the nurses might take on a coordinating or 'leadership' role. It is interesting that implementing the nested rhythms of CPR depends upon an asymmetric division of labour within the implementing team, with a focal authority being needed to coordinate and drive the protocol forward, and that the trainers are looking specifically for this to emerge. In the absence of a strong leader emerging from the ranks of the trainee nurses, it is easy to see how the AED can assume authority and influence the team to an undue degree, which was noticed by nurses and the nurse trainers as the simulations progressed. In one postsession debrief the group of trainee nurses expressed concern that they were still not smoothly implementing the CPR rhythm, even after getting couple of simulations under their belt. After some discussion, the trainers commented that the trainees were focussing too closely on the instructions of the AED, which interfered with them achieving a smooth choreography. The trainers recommended that the trainees perform the next resuscitation without using the AED, so that they could focus more on an uninterrupted delivery of the underlying protocol. When they did this, the team of trainees were more satisfied with the outcome.

We observed a number of discussions where the extent and limits to the nurse's authority were foregrounded and treated as opportunities for pedagogy by the nurse trainers, particularly in the briefings before and after each simulation. For example, when briefed for the very first scenario, the trainees were instructed not to 'act beyond their capacity as 
student nurses', but working out where precisely these boundaries lay became a pressing issue when performing resuscitation prior to the arrival of more experienced help. During one session, the nurses, working alone, debated as to whether they were permitted to use an 'epi pen' to reverse suspected anaphylaxis, which then became a topic for discussion in the post-session debriefing. In this discussion, the nurse trainers pointed out that, throughout their career, the extent of their authority would expand, but would always also remain bounded, and that recognising and managing those boundaries is an important capacity for a nurse to possess. Moreover, these discussions and observations touched upon how there are many 'grey areas' and inconsistencies, such as where nurses are permitted to conduct a procedure in one Hospital Trust, but not another. In all of these examples we see various relational aspects of the nurses' authority - how geography, personal development, and circumstances may act to grant or restrict nurses' capacity to act in a given situation.

This leads us to a seemingly paradoxical situation where a depth of experience and new types of expertise are needed to use Smart Devices effectively, yet such devices are typically aimed at enabling tasks to be conducted by less experienced personnel, whose capacity to make judgements about the instructions from automated devices is still being formed. While one might accept there is always a trade-off in using Smart Devices (e.g. earlier defibrillation versus less rigorous (PR), there are also legitimate concerns as to the sorts of shifts in competency that widespread and indiscriminate use may bring about.

\section{Conclusions}

Here we spell out some considerations arising from our study for nurse training, technology design and for the CPR protocol itself. This paper has explored the complicated ways human authorities and autonomies are increasingly influenced by the Smart Devices they use. Clearly the nurses (and even senior nurses) are susceptible to the AED which may lead to a disruption to the time-critical and multiple rhythms of CPR, and thus less effective CPR being delivered. Our problem is that machines acquire their international resources by their design - and people through experience. So how can it be possible to design machines and training so that inappropriate (or even dangerous) interactional asymmetries are avoided? How can we ensure, through design, that the 'hands off' time away from a patient is kept to a minimum?

Examples of the ways that people adapt to using peremptory devices can be found in literatures that look at alerts and notifications, For example, Iqbal (2010) and Licoppe (2010), show how peoples' choice of musical ringtones is, in part, a way of softening the imperative nature of this particular notification, and exists as part of a wider set of strategies for rendering the call as potentially ignorable. Such discretionary practices can be harder to work heavily institutionalised settings such as medicine, where issues of liability and safety mitigate towards immutable, non-personalised controls. On the other hand, alarms on pieces of equipment, such as respirators, sometimes do have tailorable ranges, which are adjusted to match the experience of the duty nurse as much as the condition of the patient (Randell 2004). Below we explore some of the possibilities for fostering an environment and practices aimed at better calibrating responses towards assertive Smart Devices. 


\subsection{Nurse Training}

An important lesson from this study would be to consider how we can adapt nurse training to be more sensitive to encounters with authoritative devices in practice. It would be possible to be more explicit about some of the dangers of using an AED device, particularly when it may be ignored legitimately and the importance of maintaining 'hands-on' time irrespective of the device instructions. A trickier question is how we help nurses more generally cope with Smart Devices of varying types that they may encounter without having any prior experience. Key to this is equipping nurses with the appropriate interactional resources - but this too runs into the problem that these often depend on extensive experience to acquire.

We suggest that training should help develop active reflective practices that encourage a critical stance towards Smart Devices as they are used in real life clinical practice. This could be done by developing training materials which show practitioners can stay in control in their interaction with the devices, both by demonstrating interactional cues they may be susceptible to, and moments where it is legitimate to ignore the advice of a device. The mix of actors present varied across the training simulations we observed such that trainees either experienced situations by themselves, or with varying interactional asymmetries in relation to the other actors who happened to be present. This created a number of learning opportunities for the trainees. In the situations when the team of trainee nurses performed alone, before senior help had arrived, they were able to experience and question the boundaries of their own authority (e.g. whether or not they were 'allowed' to administer an epi-pen). On another occasion, when the trainees practiced delivering CPR without the assistance of the AED, they were able to hone their performance without feeling compelled to adhere to the AED's staccato interpretation of the protocol. Finally, in the simulation involving the 'doctor' the trainees were exposed to an alternative interactional asymmetry to their own, and given an object lesson in how to exert authority over the AED. Whilst these variations occurred naturally as the sequence of simulations unfolded, they point to ways that simulation scenarios may be more explicitly designed to include exposure to different authority-relationships to aid nurses to develop their own interactional resources for dealing with those authorities.

\subsection{Design of the Technologies}

One might see the authority of the Smart Devices as emerging from characteristics of the devices themselves, by virtue of their supposed fidelity to the protocol and assertive interjection of beeps and use of a loud authoritative voice. And while we agree that these factors play a part, it is also the case that the AED's authority is in part granted by the nurses who orient to its advice and direction as legitimate, and ratify this by responding to the devices instructions. We also saw an occasion where the Smart Watch was disruptive to the CPR routine at moments when the nurses inappropriately followed its advice to the letter. Despite these orientation of the nurses, the AED's authority is actually illegitimate in the hospital situation, since it was actually designed for lay use in public spaces, and the versions of the CPR protocol it contains is 'watered down' to ensure that it would be manageable by a lay person ${ }^{5}$.

\footnotetext{
${ }^{5}$ There is an ever-present tension and trade-off between the 'purity' of the CPR protocol and the practicalities of its application (Timmermans, 2010).
} 
It is worth drawing a comparison between Smart Devices that actively encourage compliance with a protocol, and more neutral or passive ways of conveying the protocol, such as flow charts and documentation. This brings us back to the literature we outline earlier in relation to autonomy and clinical protocols. Although we accept that, as with paper protocols, that Smart Devices do not present an irresistible and totalising threat to clinical autonomy (Timmermans et al., 2004), our example shows that for certain staff at a particular moment in their professional development, there may be greater susceptibility to authoritative devices. Moreover, there is a qualitative difference between Smart Devices and (paper) protocols. Issue of autonomy and authority seems more salient for Smart Devices as they give an authoritative voice to the protocol, animating them and bringing them to life deep within the moments of clinical practice. It is as if the protocol, after playing a supporting role for many years, now wants to take the lead. One design approach to address these problems might be to think about how to adapt the AED to different contexts. So, while the timing and pace of the AED voice were designed to introduce intentional pauses for lay people, the timing and pace of the instructions could be adjusted for different users to enable more 'hands on' time for different users with varying degrees of expertise.

Another approach may be to consider more carefully the phrasing and use of interactional cues used to give instructions, such as voices, bleeps employed by the AED, as well as visual imperatives, such as the counter employed by the Smart Watch. While we saw the doctor overruling the AED, this took effort and added tension to the situation, since the device still emitted potent cues that had to be ignored. Clearly the authoritativeness of the device becomes itself a design consideration and questions arise at when the device should borrow human authoritative traits, such as an authoritative voice, and on what occasions should it employ a more neutral or passive interactional style. Drawing on our interactional analysis, then less authoritative machine utterances would be ones that that did not necessarily mandate compliance as the next legitimate turn. A further possibility is that, whilst the devices in this study are configured to always take a 'leader stance', it may be possible to introduce controls that allow the device to be 'nudged' (perhaps at different moments during the resuscitation) into changing the characteristics of its interactions towards so that it exhibits something closer to a 'follower stance' (Fairhurst 2008).

\subsection{Design of the Protocol}

In a cardiac resuscitation there are differences in the protocols utilised with and without the $A E D$, and both require establishment of authority to steer the team in a common purpose. Novices in this situation will be prone to deferring to their 'authority'- either guidance by the team leader (expert doctor) or the voice of the AED. There is a clear protocol when there is no AED present. There is a clear protocol associated with the AED itself. What our findings show is that there is no available protocol to combine professional expertise with the use of an AED, and this leads to confusion over which authority novices should follow. The CPR guidelines do exhort to 'minimise interruptions' when an AED is being used, but how far doing so entails disregarding the AED's instructions is much less clear. Our observations reveal how significant this gap can be for some inexperienced staff. So it would seem helpful for the CPR guidelines themselves to offer much more detailed advice about dealing with assistive devices. Earlier we saw that applying a guideline is a matter for professional expertise in fitting the regularities of the guideline to the contingencies of real 
world practice (Timmermans and Berg 1997). It is possible that when embodied in devices, and actively delivered that this type of work becomes more of an uphill struggle. Thus it may be highly valuable to make explicit and assist the development of these second-order practices - practices that are arguably just as crucial to the interpretation of active Smart Devices as they are in the performance of passive paper-based protocols.

\section{Acknowledgements}

This work was supported in part by EU FP7 FET SmartSociety project (http://www.smartsociety-project.eu/) under the Grant agreement n.600854. It was also supported by the CLEAR (Comparison of Learning Event and Actual Resuscitation) project which was funded by a small grant from the Web Science Institute (University of Southampton) Stimulus Fund in Sept 2014.

\section{References}

Ammenwerth, Elske; Petra Schnell-Inderst; Christof Machan; and Uwe Siebert (2008). The Effect of Electronic Prescribing on Medication Errors and Adverse Drug Events: A Systematic Review. Journal of the American Medical Informatics Association, vol. 15, no. 5, pp. 585-600.

Andersen, Peter Oluf; Michael Kammer Jensen; Anne Lippert; and Doris $\varnothing$ stergaard (2010). Identifying non-technical skills and barriers for improvement of teamwork in cardiac arrest teams. Resuscitation, vol. 81, no. 6, pp 695-702.

Bardram, Jakob E. (2000). Temporal Coordination-On Time and Coordination of Collaborative Activities at a Surgical Department. Computer Supported Cooperative Work (CSCW), vol. 9, no. 2, pp 157-187.

Bardram, Jakob E.; and Claus Bossen (2005). A web of coordinative artifacts: collaborative work at a hospital ward. GROUP'05. Proceedings of the 2005 international ACM SIGGROUP conference on Supporting group work, Florida, USA, 6- 9 November 2005. New York: ACM Press, pp. 168-176.

Cabitza, Federico; and Carla Simone (2009). Active artifacts as bridges between context and community knowledge sources. $C \& T^{\prime} 09$. Proceedings of the fourth international conference on Communities and technologies, Philadelphia, USA, 25- 27 June, 2009. New York: ACM Press, pp. 115-124.

Castelao, Ezequiel Fernandez; Sebastian G Russo.; Martin Riethmuller; and Margarete Boos (2013). Effects of team coordination during cardiopulmonary resuscitation: A systematic review of the literature. Journal of Critical Care, vol. 28, no. 4, pp. 504-521.

Cicourel, Aaron V (1990). The integration of distributed knowledge in collaborative medical diagnosis. In J. Galegher; R. E. Kraut; and C. Egido (eds): Intellectual Teamwork: Social 
and Technological Foundations of Cooperative Work. Hillsdale, New Jersey: Laurence Erlbaum Associates.

Cooper, Simon; and Alan Wakelam (1999). Leadership of resuscitation teams: 'Lighthouse Leadership'. Resuscitation, vol. 42, no. 1, pp. 27-45.

Cummings, Kathlene; and Ryan McGowan (2011). Smart infusion pumps are selectively intelligent. Nursing, vol. 41, no. 3 pp. 58-9.

Donchin, Anne (1995). Reworking autonomy: Toward a feminist perspective. Cambridge Quarterly of Healthcare Ethics, vol 4, no. 1, pp. 44-55.

Drew, Paul; and John Heritage (1992). (Eds.) Talk at Work. Cambridge and New York: Cambridge University Press.

Elgin, Krissa; and Cassandra Bergero (2015). Technology and the Bedside Nurse: An Exploration and Review of Implications for Practice. Nursing Clinics of North America, vol. 50 no. 2 , pp. 227-239.

Epling, Michael; Stephen Timmons; and Heather Wharrad (2003). An educational panopticon? New technology, nurse education and surveillance. Nurse Education Today, vol. 23, no. 6, pp. 412-418.

Fairhurst, Gail T. (2008). Discursive leadership: A communication alternative to leadership psychology. Management Communication Quarterly, vol. 21, no. 4, pp. 510-521.

Fitzpatrick, Geraldine; and Ellingsen Gunnar (2013). A review of 25 years of CSCW research in healthcare: contributions, challenges and future agendas. Computer Supported Cooperative Work (CSCW), vol. 22, no. 4-6, pp. 609-665.

Jefferson, Gail (1972). Side sequences. In D.N. Sudnow (ed.): Studies in social interaction. New York, NY: Free Press, pp. 294-33.

Grünerbl, Agnes; Gerald Pirkl; Mark Weal; Mary Gobbi; and Paul Lukowicz. (2015). Monitoring and enhancing nurse emergency training with wearable devices. ISWU'15. Adjunct Proceedings of the 2015 ACM International Joint Conference on Pervasive and Ubiquitous Computing and Proceedings of the 2015 ACM International Symposium on Wearable Computers, Osaka, Japan, 7-11 September 2005. New York: ACM Press, pp. 1261-1267.

Heath, Christian; John Hindmarsh; and Paul Luff (2010). Video Analysis and Qualitative Research. London: Sage

Iqbal, Shamsi T.; and Horvitz. Eric (2010). Notifications and awareness: a field study of alert usage and preferences. CSCW'10. Proceedings of the 2010 ACM conference on Computer supported cooperative work, Savannah, USA, 6 - 10 February 2010. New York: ACM Press, pp. 27-30. 
Kent, Bridie; Bernice Redley; Nilmini Wickramasinghe; Lemai Nguyen; Nyree J Taylor; Hoda Moghimi; and Mari Botti (2015). Exploring nurses' reactions to a novel technology to support acute health care delivery. Journal of Clinical Nursing, vol. 24, nos. 15-16, pp. 2340-2351.

Kusunoki, Diana S.; and Aleksandra Sarcevic (2015). Designing for Temporal Awareness: The Role of Temporality in Time-Critical Medical Teamwork. CSCW'15. Proceedings of the 18th ACM Conference on Computer Supported Cooperative Work \& Social Computing, Vancouver, Canada, 14 - 18 March 2015. New York: ACM Press, pp. 1465-1476.

Lee, Soyoung; Tang, Charlotte; Park, Sun Young; and Chen, Yunan. (2012) Loosely formed patient care teams: communication challenges and technology design. CSCW'12. Proceedings of the ACM 2012 conference on Computer Supported Cooperative Work, Seattle, USA, 11-15 February 2012. New York: ACM Press, pp. 867-876.

Licoppe, Christian (2010). The "crisis of the summons": A transformation in the pragmatics of "notifications," from phone rings to instant messaging. The Information Society. vol. 26 , no. 4 , pp. 288-302.

Manias, Elizabeth; and Annette Street (2000). Legitimation of nurses' knowledge through policies and protocols in clinical practice. Journal of Advanced Nursing. vol 32, no. 6, pp. 1467-1475.

McCarthy, Eunice. (1996) Culture, Mind and Technology: Making a Difference. In K.S. Gill (ed.): Human Machine Symbiosis. Springer: London, pp. 143-176.

McKay, Andrew; Susanna. T. Walker; Stephen. J. Brett; Charles Vincent; and Nick Sevdalis (2012). Team performance in resuscitation teams: Comparison and critique of two recently developed scoring tools. Resuscitation, vol. 83, no.12, pp. 1478-1483.

McLanders, Mia; Penelope Sanderson; and Helen Liley. (2015) Neonatal resuscitation guidelines: How human factors can improve practice. In G. Lindgaard and M. Moore (eds): IEA'15. Proceedings 19th Triennial Congress of the IEA, Melbourne, Australia, 9-14 August, 2015. International Ergonomics Association, no. 1086

MacDonald, Chris. (2002) Nurse autonomy as relational. Nursing ethics, vol 9, no. 2, pp. 194201.

Meredith, Christina; and Judy Edworthy (1995). Are there too many alarms in the intensive care unit? An overview of the problems. Journal of Advanced Nursing, vol. 21, no. 1, pp. 15-20.

Miller, Daniel; Cameron Crandall; Charles Washington; and Steven McLaughlin (2012). Improving teamwork and communication in trauma care through in situ simulations. Academic Emergency Medicine, vol 19, no. 5 pp. 608-612. 
Authority as an interactional achievement

Monsieurs, Koenraad G; Catherine Vogels; Leo L. Bossaert; Philippe Meert; and Paul A. Calle (2005). A study comparing the usability of fully automatic versus semi-automatic defibrillation by untrained nursing students. Resuscitation, vol 64, no. 1, pp. 41-47.

Monger, Ellie; and Mary Gobbi (2006). Protocol for Research and Education/Ethics into Virtual Interactive Practice.(PREVIP) Version 4. Unpublished Protocol University of Southampton.

Orwat, Carsten; Andreas Graefe; and Timm Faulwasser (2008). Towards pervasive computing in health care-A literature review. BMC Medical Informatics and Decision Making, vol. 8, no. 1.

Pine, Katie (2012). Fragmentation and choreography: caring for a patient and a chart during childbirth. CSCS'12. Proceedings of the ACM 2012 conference on Computer Supported Cooperative Work, Seattle, USA, 11-15 February 2015. New York: ACM Press, pp. 887896.

Randell, Rebecca (2004) Accountability in an alarming environment. CSCW'04. Proceedings of the 2004 ACM conference on Computer supported cooperative work, Chicago, USA, 610 November 2004. New York: ACM Press, pp. 125-131.

Reddy, Madhu C; Paul Dourish; Wanda Pratt (2006). Temporality in medical work: Time also matters. Computer Supported Cooperative Work (CSCW). vol. 15, no. 1, pp. 29-53.

Resuscitation Council (UK) (2015). Resuscitation Guidelines. Resuscitation Council, United Kingdom https://www.resus.org.uk/statements/rc-uk-resuscitation-guidelines-2015published/

Roberts, Nicole K.; Reed G. Williams; Cathy J.Schwind; John A.; Sutyak; Christopher McDowell; David Griffen; Jarrod Wall; Hilary Sanfey; Audra Chestnut; Andreas H Meier.; Christopher Wohltmann; Ted R Clark.; and Nathan Wetter (2004). The impact of brief team communication, leadership and team behavior training on ad hoc team performance in trauma care settings. The American Journal of Surgery, vol. 207, no. 2 pp. 170-178.

Rose, Jim. (2015). Article: This autonomous medical robot can assist nurses. http://www.extremetech.com/extreme/207130-terapio-autonomous-medical-robotcan-assist-nurses

Rothschild, Jeffrey M.; Carol A. Keohane; Sarah Thompson; and David W. Bates (2003). Intelligent intravenous infusion pumps to improve medication administration safety. In AMIA'03. AMIA Annual Symposium Proceedings, Washington, USA, 16-20 November 2013. American Medical Informatics Association, pp. 992. 
Sarcevic, Aleksandra; Ivan Marsic; Lauren J. Waterhouse; David C. Stockwell; and Randall S. Burd (2011). Leadership structures in emergency care settings: A study of two trauma centers. International Journal of Medical Informatics, vol. 80, no. 4, pp. 227-238.

Schenarts, Paul J. (2007) Incorporating leadership training, a horizontal approach to resuscitation and performance feedback, into advanced life support. Critical care medicine. vol. 35, no. 7, pp. 1781-1782.

Schneider, Barbara. (2017) Power as an Interactional Accomplishment: An Ethnomethodological Perspective on the Regulation of Communicative Practice in Organisations. In Zachry, Mark, and Thralls, Charlotte (eds): Communicative practices in workplaces and the professions: Cultural perspectives on the regulation of discourse and organizations. Routledge.

Smith, Roger J.; Bernadette B Hickey; and John D. Santamaria (2011). Automated external defibrillators and in-hospital cardiac arrest: patient survival and device performance at an Australian teaching hospital. Resuscitation, vol. 82, no. 12, pp. 1537-1542.

Timmermans, Stefan; and Emily S. Kolker (2004). Evidence-Based Medicine and the Reconfiguration of Medical Knowledge. Journal of Health and Social Behavior. Extra Issue: Health and Health Care in the United States: Origins and Dynamic, vol. 45, pp. 177-193.

Timmermans, Stefan; and Marc Berg (1997). Standardization in action: achieving local universality through medical protocols. Social studies of science, vol. 27, no. 2, pp. 273305.

Timmermans, Stefan. (2010) Sudden Death and the Myth of CPR. Philadelphia: Temple University Press.

Wacker, Johannes; and Michaela Kolbe (2014). Leadership and teamwork in anaesthesiaMaking use of human factors to improve clinical performance. Trends in Anaesthesia and Critical Care, vol 4, no. 6, pp. 1200-205.

Weston, Marla J (2008). Defining control over nursing practice and autonomy. Journal of Nursing Administration, vol. 38, no. 9, pp. 404-408.

Yamada, Nicole K; Kim A Yaeger;. And Louis P Halamek (2015). Analysis and classification of errors made by teams during neonatal resuscitation. Resuscitation, vol.96, pp. 109-113. 